\title{
Neurocutaneous melanosis
}

\section{Melanose neurocutânea}

\section{Roselane Sampaio de Oliveira ${ }^{1}$, Ana Paula Rangel de Carvalho', Fábio Noro², Alessandro Severo Alves de Melo, Ruy Monteiro ${ }^{4}$, Rodrigo Guimarães ${ }^{4}$, José Alberto Landeiro ${ }^{5}$}

${ }^{1}$ Resident Physician in the Radiology Department at Barra D'or Hospital, Rio de Janeiro RJ, Brazil;

${ }^{2}$ Radiologist at Rede Labs D'or, Rio de Janeiro RJ, Brazil;

${ }^{3}$ Adjunct Professor of the Department of Radiology at Universidade Federal Fluminense, Niteroi RJ, Brazil;

${ }^{4}$ Neurosurgeon at Rede Labs D'or, Rio de Janeiro RJ, Brazil;

${ }^{5}$ Adjunct Professor of the Department of Neurosurgery at Universidade Federal Fluminense, Niteroi RJ, Brazil.

Correspondence: Roselane Sampaio de Oliveira; Avenida Ayrton Senna 2541, Barra da Tijuca; 22775-002 Rio de Janeiro RJ - Brazil; E-mail: roselanesam@yahoo.com.br

Conflict of interest: There is no conflict of interest to declare.

Received 14 December 2011; Received in final form 16 July 2012; Accepted 23 July 2012

Neurocutaneous melanosis (NCM) is a rare phakomatosis that was first observed in 1861, in a 14-year-old girl born with a congenital nevus and suffering from mental retardation ${ }^{1}$. The pathogenesis of NCM is believed to result from an error in the morphogenesis of the neuroectoderm during embryogenesis ${ }^{1,2}$.

In this paper, we report a case of this phakomatosis, describe, and discuss the findings from cranial computed tomography (CT) scan and magnetic resonance imaging (MRI).

\section{CASE DESCRIPTION}

Our patient was a 29-year-old woman with a four-month history of sporadic seizures and a giant congenital melanocytic nevus on the torso (Fig 1A).

CT scan and MRI (Fig 1B and 1C) of the central nervous system (CNS) showed several lesions, which were later biopsied (Fig 1D). Histopathological analysis revealed a melanoma (Fig 1E), and the patient was then subjected to complementary examinations in order to detect the primary lesion, but without success.

The patient suffered brain death 21 days after hospital admission.

\section{DISCUSSION}

The diagnostic criteria for NCM were described by Fox and later reviewed by Kadonaga and Frieden ${ }^{3}$. They include the following:

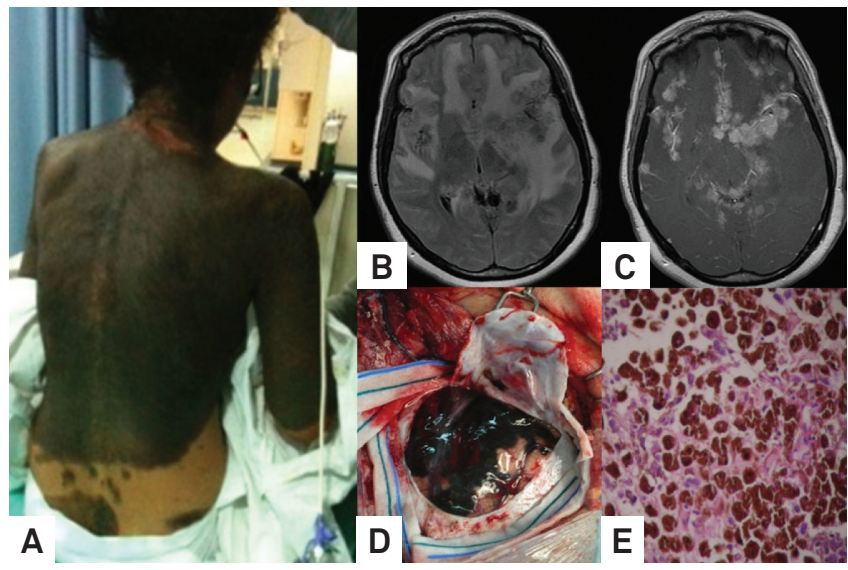

Fig 1. (A) Giant melanocytic nevus on the torso, extending to the upper limbs and neck. (B) Axial FLAIR showing highsignal intensity lesions, along with edema, thus reinforcing the hypothesis of evolvement to melanoma. (C) Post-contrast axial T1-weighted image showing lesions with significant enhancement. (D) During surgical biopsy on the NCM, large pigmented lesions were observed at the leptomeninges. (E) Photomicrograph of a leptomeningeal sample (x200 magnification; hematoxylin-eosin staining) showing proliferation of pigmented melanocytic cells.

giant congenital nevus (usually larger than $20 \mathrm{~cm}$ in adults and between 6 to $9 \mathrm{~cm}$ in children) or multiple congenital nevi (more than three) in association with meningeal melanosis or CNS melanoma;

absence of cutaneous melanoma, except in cases of histologically benign meningeal lesions; 
- absence of meningeal melanoma, except in cases of benign dermatological lesions.

The symptoms of NCM include seizures, neurological focal deficit and psychiatric disorders ${ }^{1,4}$. The CNS may be compromised through parenchymatous or leptomeningeal lesions, melanosis (aggregation of benign melanocytic cells) or melanomas ${ }^{1,4}$.

The lesions shown on CT scans may be isodense or hyperdense. Melanomas (parenchymal or leptomeningeal form) and leptomeningeal melanosis usually show enhancement after contrast administration ${ }^{4}$.

On MRI, T1-weighted sequences show hyperintense parenchymal lesions, while leptomeningeal lesions may be isointense or hyperintense. After contrast administration, parenchymal melanosis does not commonly show enhancement in T1, while leptomeningeal lesions (melanosis and melanoma) show diffuse enhancement ${ }^{1,4}$.

On T2-weighted sequences, lesions usually present a low or intermediate signal. It should be noted that lesions present high signal intensity in FLAIR (fluid-attenuated inversion recovery) $)^{1,4}$.

It is well known that the signs that indicate melanoma instead of benign melanocytic lesions are mass effect, edema, hemorrhage and necrosis ${ }^{1,4}$.

Leptomeningeal lesions associated with edema were observed at CT scan and MRI in our patient, indicating possible melanoma, as later confirmed by the histopathological analysis. Even though the image characteristics may indicate benign melanocytic lesions or melanoma, the prognosis is better correlated with the onset of neurological symptoms ${ }^{4}$.

The treatment is palliative and includes systemic and intrathecal chemotherapy and radiotherapy, as well as ventriculoperitoneal shunt (with filter) in cases of hydrocephaly. In addition, regardless of the treatment, the prognosis is bad $\mathrm{ba}^{4}$.

In conclusion, although it is a rare entity, NCM should be investigated among patients presenting central neurological symptoms associated with congenital melanocytic nevus.

\section{References}

1. Smith AL, Rushing EJ, Smirniotopoulos JG. Pigmented lesions of de central nervous system: radiologic-pathologic correlation. Radiographics 2009;29:1503-1524.

2. Barkovich AJ. Pediatric neuroimaging. Fourth edition. Philadelphia: Lippincott Williams \& Wilkins, 2005. p. 487-488.
3. Kadonaga JN, Frieden IJ: Neurocutaneous melanosis: definition and review of the literature. J Am Acad Dermatol 1991;24:747-755.

4. Osborn AG, Blaser SI, Salzman KL, Katzman GL, Provenzale J, Castillo M. Diagnostic imaging: Brain. Salt Lake City: Amirsys, 2004:|-1-116-|-1-119. 